bles) y su depósito en los túbulos renales. El diagnóstico puede establecerse realizando análisis de sangre, donde encontramos elevación de urea y creatinina, y confirmarse con una analítica de orina donde observamos un $\mathrm{pH}$ menor de 6 , hematuria y depósito de cristales de $\mathrm{N}$-acetilsulfadiazina en el sedimento. En la ecografía renal podemos observar imágenes ecogénicas compatibles con nefrolitiasis o, más frecuentemente, hallazgos normales. El cuadro clínico puede aparecer tanto al inicio (más frecuente) como a lo largo del tratamiento y suele caracterizarse por dolor abdominal tipo cólico, náuseas acompañadas o no de vómitos, hematuria y, si persiste, oliguria e insuficiencia renal aguda (9). El tratamiento consiste en la hidratación adecuada manteniendo diuresis diaria de 2 o más litros y conseguir mantener un $\mathrm{pH}$ urinario mayor de 7 . En los casos graves (acidosis severa, hipercaliemia y uremia severa) con fallo renal agudo, estaría indicado el tratamiento con hemodiálisis (10). No existen estudios que demuestren la necesidad de suspender el tratamiento con sulfadiazina, aunque desde el punto de vista práctico, se aconseja disminuir dosis o incluso cambiar la terapia iniciada que incluye la sulfadiazina por la combinación clindamicina-pirimetamina. Una vez resuelto el episodio de cristaluria, nefrolitiasis e insuficiencia renal, si se ha producido, y corregido los parámetros analíticos, la reintroducción de la sulfadiazina debe ser evaluada individualmente.

Podemos concluir que la aparición de depósito de cristales, nefrolitiasis y/o insuficiencia renal, al inicio o durante el tratamiento con sulfadiazina de la TC en los pacientes con sida, no son infrecuentes y deben ser tenidos en cuenta dado que podemos aplicar medidas preventivas. Estas medidas consisten en una adecuada hidratación para conseguir diuresis de 2-3 litros diarios y mantener un $\mathrm{pH}$ urinario mayor de 7 . Con ello podemos evitar, prácticamente en la totalidad de casos, la aparición de estos efectos adversos y de sus posibles complicaciones. No está claramente demostrado que sea necesaria la suspensión y/o la sustitución de sulfadiazina-pirimetamina por la combinación clindamicinapirimetamina para continuar el tratamiento de la TC durante 6-8 semanas. Existen estudios recientes donde no se observaron datos que sugiriesen contraindicación de continuar tratamiento con sulfadiazina una vez resuelto el episodio. Sin embargo, la experiencia clínica aconseja la suspensión del tratamiento con sulfadiazina, y su sustitución, en caso de aparecer las complicaciones descritas, y valorar individualmente la reintroducción de la sulfadiazina, una vez resueltas dichas complicaciones, en los pacientes seropositivos con toxoplasmosis cerebral.

M. Solano Remírez, A. S. Oliván Ballabriga, G. M. da Costa Rodríguez, M. T. Álvarez Frías, C. González Arencibia

Servicio de Medicina Interna. Hospital de Navarra. Pamplona

1. Clumeck N. Some aspects of the epidemiology of toxoplasmosis and Pneumocystis in AIDS in Europe. Eur J Clin Microbiol Infect Dis 1991; 10: $177-8$

2. Porter S, Sande M. Toxoplasmosis of the central nervous system in the acquired inmunodeficiency syndrome. N Engl J Med 1992; 327: 16438.

3. Ciricillo $\mathrm{S}$, Rosemblum M. Use of CT and MR imaging to distinguish intracranial lesions and to define the need for biopsy in AIDS patients. J Neurosurg 1990; 73: 720-4.

4. Leport C, Chêne G, Morlath Ph, et al. Pyrimethamine for primary of toxoplasmic encephalitis in patients with human immunodeficiency virus infection: a double-blind, randomized trial. J Infect Dis 1996; 173: $91-7$.

5. Miro J, López J, Podzamczer D, et al. Discontinuation of primary or secondary Toxoplasma gondii prophylaxis is safe in VIH-1-infected patients after immunological recovery with HAART. Final results of the
GESIDA 04/98-B study. 40th ICAAC. Toronto, septiembre 2000 (presentación L-16).

6. Katlama C, De Wit S, O'Doherty E, et al. Treatment of toxoplasmosis in AIDS patients. Clin Infect Dis 1996; 22: 268-75.

7. Wong S, Israelski D, Remington J. AIDS-associated toxoplasmosis. En: Sande M, Volberding P (eds.). The medical management of AIDS. 4. ${ }^{\mathrm{a}}$ ed. Philadelphia: WB Saunders 1995: 460-93.

8. Dannemann B, McCuthan J, Israelski D, et al. Treatment of toxoplasmic encephalitis in patients with AIDS: A randomized trial comparing pyrimethamine plus clindamycin to pyrimethamine plus sulphadiazine. The California Collaborative Treatment Group. Ann Intern Med 1992; 116: 33-43.

9. Becker K, Jablonowski H, Haussinger D. Sulfadiazine-associated nephrotoxicity in patients with adquired immunodeficiency syndrome. Medicine-Baltimore 1996; 75: 185-94.

10. Don B, Rodriquez RA, Humphreys MH. Acute renal failure associated with pigmenturia or crystal deposits. In: Schrier RW, Gottschalk CW, editors. Diseases of the kidney. 6th ed. Boston: Little, Brown, 1997.

\section{Crisis convulsiva por bupropion}

\section{Sr. Director:}

Dentro de los tratamientos empleados para la deshabituación al tabaquismo, en los últimos años ha demostrado ser eficaz un medicamento (bupropion) ampliamente empleado en el tratamiento de los trastornos depresivos (1). Es conocido y aceptado que los estados de ánimo y del humor producen importantes efectos en la motivación para consumir nicotina. Se ha visto que los fumadores son más propensos a presentar síntomas propios de los trastornos de afectividad que los no fumadores (2). Los síntomas de dependencia a la nicotina se correlacionan con la intensidad de síntomas en la esfera de la afectividad, por lo que el estado de ánimo bajo ó negativo conlleva a una mayor disponibilidad para comenzar a fumar y mayor dificultad para el abandono del hábito tabáquico, pudiendo estar relacionado con alteraciones en la actividad dopaminérgica cerebral (4). Clásicamente, los derivados de la nicotina, en forma de chicles ó de parches, han demostrado su eficacia en la deshabituación tabáquica multiplicando las tasas de abandono del hábito conseguidas con placebo, aunque en un porcentaje no despreciable de estos, vuelven a recaer. Cada año, aproximadamente unos 20 millones de fumadores estadounidenses intentan dejar el hábito, pero sólo un $6 \%$ de los que lo intentan, consiguen, a largo plazo, abandonarlo. En un estudio a doble ciego y controlado con placebo en 1999, DE Jorenby y cols. demostraron que el tratamiento con bupropion solo ó asociado a parches de nicotina alcanzó, a largo plazo, tasas de deshabituación significativamente superiores a las obtenidas con parches de nicotina solos o placebo, siendo las tasas de abstinencia con terapia combinada superiores a la conseguida con bupropion solo, pero no alcanzando éstas una significación estadística (3). La mayoria de los efectos adversos del bupropion son, en general, de carácter leve, y dosis-dependientes (cefalea, insomnio, sequedad de boca...); entre los de carácter grave destaca, por su importancia clínica, las crisis convulsivas.

Presentamos el caso de una mujer de 20 años de edad en cuyos antecedentes personales podemos destacar un hábito tabáquico importante (más de 20 cigarrillos al día) en tratamiento de deshabituación desde hace 40 días con bupropion 1 comprimido/12 h; no traumatismo craneoencefálico previo. Los antecedentes familiares carecen de interés. Presentó episodio de pérdida de conciencia brusco con caída al suelo, seguido de movimientos convulsivos tónico-clónicos generalizados asociado a mordedura de 
lengua, pérdida de control de esfínteres y confusión post-ictal, con posterior recuperación, lenta, de la actividad consciente. Refiere amnesia de los hechos sin precisar el tiempo de duración del episodio. Posteriormente presentó otros dos episodios convulsivos generalizados de menos de cinco minutos de duración de características similares al referido. En la exploración física tiene TA $120 / 70$ y T ${ }^{a} 36^{\circ}$; está perceptiva, orientada y colaboradora; no presenta signos meníngeos; fondo de ojo y resto de pares craneales normales; pequeña herida por mordedura en ambos lados de la lengua; resto de examen neurológico y general normal. La analítica de sangre no aporta datos relevantes. ECG, radiografía de tórax, EEG, TAC craneal y RMN craneal son normales. No presentó nuevos episodios de convulsiones ni precisó tratamiento anticonvulsivante. En revisiones posteriores se evidenció la ausencia de nuevos episodios convulsivos. Dada la normalidad del examen clínico, exploraciones complementarias y la evolución clínica se concluyó que dichas crisis convulsivas fueron (presumiblemente, teniendo en cuenta que se han descrito como reacciones adversas en pacientes en tratamiento con bupropion) por la toma de dicho fármaco.

La dosis recomendada de bupropion para la deshabituación tabáquica, en los adultos, es de $150 \mathrm{mg}$ una vez al día durante seis días y posteriormente dos veces diarias. El tratamiento se debe mantener durante 7-9 semanas; si el paciente sigue fumando deberá suspender el tratamiento. El bupropion tiene una estructura monocíclica sin semejanza a otros antidepresivos conocidos, del grupo de las aminocetonas, relacionado con las feniletilaminas (5). Si bien no ha sido aclarado su mecanismo de acción se sabe que es un inhibidor débil de la dopamina y la noradrenalina; aumenta las concentraciones de dopamina extracelular en el núcleo accumbens (una parte del sistema mesolímbico de importancia en el caso de la adicción a la nicotina); no modifica la recaptación de la serotonina y tiene un papel como antagonista no competitivo de los receptores nicotínicos a nivel músculos y ganglios autónomos. La biodisponibilidad absoluta del bupropion no ha sido determinada. Se desconoce si la proporción de dosis y el consiguiente nivel en plasma del fármaco se mantiene en el uso crónico. La absorción se lleva a cabo de forma rápida (alcanza concentración máxima en plasma a las 3 horas). Su absorción no es interferida por alimentos. Se une en un $85 \%$ a proteínas plasmáticas. Se distribuye por todos los tejidos del organismo. Se metaboliza, por óxido-reducción, en hígado por acción de las isoenzimas del complejo citocromo P450, trasformándose en tres metabolitos activos (hidroxibupropion, treohidrobupropion y eritrohidrobupropion) (6). La eliminación es fundamentalmente renal $(84 \%)$ y se lleva a cabo en dos fases. La vida media de eliminación es de 11-14 h. Difunde con facilidad al líquido cefalorraquídeo (LCR), atraviesa sin dificultad la placenta y se excreta por la leche materna. Las reacciones adversas son conocidas desde hace años y motivo de estudios, debido al amplio uso del fármaco en el mundo anglosajón en el tratamiento de los tras- tornos depresivos, sólo o asociado a otros antidepresivos (7). Podemos clasificar las diferentes reacciones adversas en orden decreciente en función de la definición de frecuencia: Frecuente $(1 \mathrm{caso} / 100$ pacientes), infrecuente (entre $1 / 100$ y $1 / 1.000) \mathrm{y}$ rara (1/1.000 pacientes). Los efectos adversos más frecuentes son el insomnio, la sequedad de boca, la anorexia y el estreñimiento. Se debe tener especial cuidado en el uso concomitante con medicamentos que interaccionen con el metabolismo del bupropion, alterando sus niveles plasmáticos, o que modifiquen sus efecto. Dado que se secreta por la leche materna, debe valorarse el riesgo-beneficio del empleo en embarazadas y/o suspender la lactancia. Una de las reacciones más importantes, y motivo de revisión en este artículo, es el riesgo de presentar crisis convulsivas, que constituyen una de las causas mas frecuentes de suspensión del tratamiento $(8,9)$. El riesgo de presentar convulsiones se relaciona con una serie de factores (Tabla I). Con la dosis habitualmente empleada en la deshabituación tabáquica de $300 \mathrm{mg} /$ día, la incidencia de aparición de convulsiones es de, aproximadamente, $0,1 \%$ (1/1.000), aumentando a $0,4 \%(4 / 1.000)$ con dosis máximas recomendadas de $400 \mathrm{mg} /$ día. En un estudio prospectivo en 3.200 pacientes tratados durante 8 semanas con dosis superiores a $450 \mathrm{mg} /$ día se observó una incidencia de convulsiones en un $0.4 \%$. Con dosis entre 450-600 mg/día la incidencia estimada de convulsiones se multiplica por diez veces (10).

Podemos concluir que la incidencia de convulsiones asociada a la toma de bupropion, en la dosis recomendada para la deshabituación del tabaco, es relativamente rara. El riesgo de desarrollar crisis convulsivas es dosis-dependiente, y se correlaciona con factores predisponentes del paciente. Situaciones favorecedoras y/o el uso concomitante de medicamentos que disminuyan el umbral de aparición convulsiones, también favorecen la aparición de las mismas. Estudios prospectivos y la experiencia clínica sugieren una serie de recomendaciones que minimicen y reduzcan el riesgo de aparición de convulsiones: La dosis diaria no debe exceder de $400 \mathrm{mg}$, debiendo administrarse dos veces al día; no exceder de $200 \mathrm{mg}$ la dosis única para evitar concentraciones máximas en sangre de bupropion y/o sus metabolitos; extremar su uso en pacientes con antecedentes de cirrosis hepática reduciendo la dosis y la frecuencia sin superar $100 \mathrm{mg}$ al día o $150 \mathrm{mg}$ cada 48 horas; y administrar con extrema precaución en pacientes con historia previa de convulsiones, trauma craneoencefálico o/y otros situaciones predisponentes ya reseñados con anterioridad a lo largo de este capítulo.

M. Solano Remírez, M. T. Álvarez Frías, C. González Arencibia, J. Gutiérrez Dubois, B. Llorente Díaz

Servicio de Medicina Interna. Hospital de Navarra. Pamplona

TABLA I

SITUACIONES FAVORECEDORAS DE CRISIS CONVULSIVAS CON LA TOMA DE BUPROPION

\begin{tabular}{ll} 
Factores personales & Situaciones clínicas \\
\hline Adicción a: & Antecedentes de TCE \\
-Opiáceos & Tumor SNC \\
-Cocaína & Cirrosis hepática severa \\
-Estimulantes & Anorexia \\
Interrupción brusca de tratamiento & Bulimia
\end{tabular}

Medicamentos con comitantes

con benzodiacepinas

Diabetes

Historia previa de crisis convulsivas

\section{Antidepresivos \\ Antipsicóticos \\ Teofilina o derivados \\ Corticoides sistémicos \\ IMAO..}

\section{Diabetes}


1. Hurt RD, Sachs DPL, Glover ED, Offord KP, Johnston JA, Dale LC, et al. A comparison of sustained-release bupropion and placebo for smoking cessation. N Engl J Med 1997; 337: 1195-1202.

2. Glasman AH, Covey LS. Smoking and affective disorder. Am J Health Behav 1996; 20: 279-285.

3. Joremby DE, Leischow SJ, Nides MA, Rennard SI, et al. A controled trial of sustained-release bupropion, a nicotine patch, or both for smoking cessation. N Engl J med 1999; 340: 685-691.

4. Clifford DB, Rutherford JL, Hicks FG, Zorumski CF. Acute effects of antidepressants on hipocampal seizures. Ann Neurol 1985; 18: 692-7.

5. E. Cárdenas, E. Caballero, J. Honorato. Nuevos Medicamentos: Bupropion. Rev Med Univ Navarra 2001; 45 (3): 51-55.

6. Goodale E, Ascher J, Batey S. Cytochrome P450 metabolism of bupropion (abstract). J Am Geriatr Soc 1999; 47: S88.

7. Ascher JA, Cole JO, Colin JN, Feghner JP, et al. Bupropion: a review of its mechanism of antidepressant activity. J Clin Psychyatry 1995; 56: 395-401.

8. Kwan AL, Meiners AP, Von Grootheest AC, Lakkerker JF. Risk of convulsions due to the use of bupropion as an aid for smoking cessation. Need Tijdsch Geneeskd 2001 ; 145(6): 277-8.

9. Johnston JA, Lineberry CG, Ascher JA, Davidson J, et al. A 102-center prospective study of seizure in association with bupropion. J Clin Psychiatry 1993; 54: 202.

10. Minie I, McMcGurk C, McClintock G, Robinson J. Seizures after Bupropion overdose. Lancet 2001; 357 (9268): 1624.

\section{Púrpura trombótica trombocitopénica como debut de un cuadro neoplásico}

\section{Sr. Director:}

Aunque es bien conocida la asociación de anemia hemolítica microangiopática y de coagulación intravascular diseminada (CID) como complicaciones de carcinomas diseminados, la asociación con la púrpura trombótica trombocitopénica (PTT) es mucho más inusual aunque está descrita en la literatura.

Presentamos el caso de una mujer de 34 años, fumadora importante, sin antecedentes de interés salvo el diagnóstico de embolismo pulmonar hacía un mes, y con tratamiento anticoagulante desde entonces, que reingresa por deterioro del estado general, disnea progresiva siendo de reposo a su llegada y pérdida de diez kilos de peso en los últimos seis meses. A la exploración física presentaba un regular estado general, febrícula y leve taquipnea. Se palpaba adenopatía supraclavicular derecha de dos centímetros y axilares homolaterales de menor tamaño, siendo el resto de la exploración anodina. En la analítica presentaba: $\mathrm{Hb}: 8,4$, plaquetas: 60.000 , LDH: 1561 , siendo el resto de los parámetros normales. Gasometría arterial: $\mathrm{pO}_{2}$ : 66, $\mathrm{pCO}_{2}: 24, \mathrm{PH}: 7,4$. RX de tórax: agrandamiento hiliar bilateral y ensanchamiento mediastínico. TAC tóraco-abdominal: adenopatías mediastínicas, axilares, periportales y retroperitoneales múltiples. En el ecocardigrama se objetivó hipertensión pulmonar ligera. Al presentar nuevamente insuficiencia respiratoria, se sospechó inicialmente nuevo episodio de tromboembolismo, que coincidiendo con la presencia de adenopatías y alteraciones hematológicas hizo pensar como primera posibilidad diagnóstica de base en un linfoma. Se realizó Biopsia de Médula ósea que mostró marcada hiperplasia de la serie roja sin evidencia de infiltración tumoral. CEA: 351,2; Ca 15,3: 129, resto de marcadores normales. Se solicitó al Servicio de Cirugía biopsia del ganglio supraclavicular para intentar filiar histológicamente el cuadro. A las 72 horas, presentó fiebre, empeoramiento de su disnea, pérdida de visión de ojo derecho, parestesias y paresia de extremidad superior derecha. Estaba ictérica, taquicárdica y taquipneica y con equimosis cutáneas múltiples. Hb: 6, plaquetas: 43.000, bilirrubina de 4,7 de predominio indirecto, LDH: 2005, haptoglobina: 6, resto de funciones hepática y renales normales. Con la sospecha de hemólisis se realizaron un test de Coombs directo e indirecto que fueron negativos y un frotis en sangre periférica que mostró abundantes equistocitos. Con estos datos de anemia hemolítica microangiopática, se inició tratamiento con esteroides e infusión de plasma fresco. El empeoramiento de su disnea y la existencia de hipertensión pulmonar nos llevó a realizar una arteriografía pulmonar, por sospecha de nuevo embolismo, que mostró un déficit importante de flujo vascular periférico, manteniendo la vascularización intacta en los troncos principales. La paciente se deterioró gravemente en las horas siguientes con estado confusional. El resultado anatomopatológico de la biopsia fue de metástasis de adenocarcinoma de células en anillo de sello, destacando el origen gástrico como primera posibilidad. La paciente falleció en las dos horas siguientes. El diagnóstico fue de adenocarcinoma metastásico de probable origen gástrico y púrpura trombótica trombocitopénica asociada.

La PTT (1) es un síndrome desencadenado por la oclusión diseminada de la microcirculación y que clínicamente se caracteriza por anemia hemolítica, trombocitopénia, síntomas neurológicos, fiebre y disfunción renal. Otras manifestaciones incluyen cuadros hemorrágicos, malestar general, naúseas y vómitos, disnea y arritmias. El diagnóstico diferencial $(2,3)$ debe hacerse fundamentalmente con la CID, la cual cursa con alteraciones de la coagulación y con el síndrome hemolítico urémico, que corresponde con la afectación local renal de dicho proceso microangiopático. En cuanto al desencadenante etiológico (4) en general afecta a personas sana, cuando es secundaria se ha relacionado con el embarazo, infecciones, colagenopatías, vacunas, anticonceptivos orales, fármacos, citostáticos, trasplante alogénico y enfermedad tumoral. En relación con los tumores, no parece estar en relación con un curso avanzado, en los casos descritos presentaba una remisión objetiva (extremadamente raro como presentación inicial de un tumor). Habitualmente se trata de adenocarcinomas fundamentalmente gástricos, colon, mama y menos con la Leucemia mieloide crónica. El pronóstico cuando existe una causa secundaria es desfavorable. El tratamiento consiste en el aporte de plasma y en la plasmaféresis (sin demora si existe clínica neurológica), siendo la tasa de respuestas en torno al $20 \%$ en los pacientes con cáncer.

\section{I. Gallegos Sancho, M. Reboredo López, R. García Cam- pelo, M. Quindós Varela , L. M Antón Aparicio}

Servicio de Oncología Médica. Complejo Hospitalario Universitario Juan Canalejo. La Coruña

1. Kwaan HC, Gordon LI. Thrombotic Microangiopathy in the Cancer Patient. Acta Haematol 2001; 106: 52-56.

2. Gordon LI, Kwaan HC. Thrombotic Microangiopathy manifesting as Thrombotic Thrombocytopenic Purpura/Hemolytic uremic.

3. Girad P, Tardy B, Page Y, Mosnier JF, Tardy-Poncet B, Bertrand JC. Purpura Thrombotique Thrombocytopénique révélant un cancer: à propos de deux cas. Presse Med 1995; 24: 175-8.

4. Gordon LI, Kwaan HC. Cancer-and Drug-Associated Thrombotic Thrombocytopenic Purpura and Hemolytic uremic Syndrome. Seminars in Hematology 1997; 34: 140-147. 